\title{
Themenheft „Alpine Hydrogeologie in Forschung und Anwendung“
}

\author{
Gerfried Winkler ${ }^{1}$ (D) $\cdot$ Nadine Göppert ${ }^{2}$
}

Eingegangen: 2. Dezember 2019 / Überarbeitet: 2. Dezember 2019 / Online publiziert: 20. Januar 2020

(c) Springer-Verlag GmbH Deutschland, ein Teil von Springer Nature 2020

Die „Alpine Hydrogeologie“ hat im letzten Jahrzehnt in der Forschung eine deutlich höhere Aufmerksamkeit als in den Jahrzehnten davor erfahren, sicherlich auch dadurch motiviert, dass Gebirgsregionen im besonderen Maße von Auswirkungen des Klimawandels betroffen sind. Der Stellenwert der Alpinen Hydrogeologie zeigte sich jüngst im Titel des „Darcy Lecturers“ Masaki Hayashi der National Groundwater Association 2018, der weltweit zum Thema „Alpine Hydrogeology: The Critical Role of Groundwater in Sourcing the Headwaters of the World" referierte. In der Zeitschrift Grundwasser war 2011 ein Editorial dem Thema „Alpine Hydrogeologie“ gewidmet.

Gebirge sind meist sehr wasserreich, da in diesen Regionen höhere Niederschlagsmengen, geringere Verdunstung und Lufttemperaturen als im umgebenden Vorland zusammenspielen. Große Wassermengen werden saisonal als Schnee und längerfristig in Form von Gletscher- und Permafrosteis zwischengespeichert. Das Abschmelzen dieser Speicher spielt nicht nur für den Abfluss in den Alpen und dem Alpenvorland eine Rolle, sondern ist auch wesentlicher Bestandteil der Grundwasserneubildung in alpinen Regionen und auch im Alpenvorland. Daher gelten die Alpen als Wasserturm Europas. In den letzten Jahrzehnten entwickelte sich jedoch durch zunehmende landwirtschaftliche und touristische Nutzung alpiner Regionen vermehrt ein Spannungsfeld zwischen nachhaltigem Nutzen und Schutz der Ressource Wasser und dem steigenden Wasserbedarf.

Verkarstungsfähige wie nicht verkarstungsfähige Festgesteine, aber auch große Lockergesteinsablagerungen und Talfüllungen bilden Grundwasserleiter, die für die Trinkwasserversorgung, aber auch für die Energiegewinnung ge- nutzt werden können. Vor allem komplexe geologische Verhältnisse und ein Austausch von Grundwässern aus mehreren Grundwasserleitern mit gänzlich unterschiedlichen hydraulischen Eigenschaften sind der Grund für immer neue Herausforderungen, sowohl in der Praxis, der angewandten Forschung als auch in der Grundlagenforschung. Hinzu kommen sich ändernde Grundwasserneubildungsverhältnisse aufgrund des Klimawandels. Viele hydrogeologische Fragestellungen in alpinen Regionen sind daher nur über interdisziplinäre Ansätze und ein solides Grundverständnis der komplexen geologischen Gegebenheiten zufriedenstellend zu beantworten.

Wir freuen uns, dass der Aufruf zu diesem Themenband eine so große Resonanz erhielt und die zahlreichen eingereichten Beiträge eine breite Themenvielfalt und Interdisziplinarität aufweisen. Das Themenheft spiegelt die Herausforderungen bei konkreten Anwendungen wider. Hierzu gehören die Verwendung von Grundwasser als Trinkwasser in Karstgebieten, die Bedeutung von inneralpinen Talfüllungen mit komplexen Randbedingungen ebenso wie die Hydrogeologie von Bergsturzmassen. Beiträge zu Grundlagenforschung und angewandter Forschung umfassen Themen zur Mobilisierung und Ausbreitung von geogenen und anthropogenen Schadstoffen, thermisches Verhalten von Grundwässern und ihre potenzielle Nutzung sowie die Charakterisierung von Grundwasserleitern in Permafrostgebieten. Neben den Beiträgen im aktuellen Themenheft erschien bereits ein Beitrag im Heft 4/2019, und zwei weitere zum Thema „Alpine Hydrogeologie“ werden im Heft 2/2020 folgen.

Gerfried Winkler

gerfried.winkler@uni-graz.at

Nadine Göppert

nadine.goeppert@kit.edu

1 Institut für Erdwissenschaften, Karl-Franzens-Universität, Graz, Österreich

2 Institut für Angewandte Geowissenschaften, Karlsruher Institut für Technologie, Karlsruhe, Deutschland 